\title{
Sp(6, R) SYMMETRY AND $\propto$-BREAKUP AMPLTTUDS OF GIANT E2 EXCITATIONS IN LIGHT NUCLEI
}

\author{
K. T. HECHT and D. BRAUNSCHWEIG ' \\ Physics Department, University of Michigan, Ann Arbor, Michigan 48109, USA
}

Received 12 September 1977

\begin{abstract}
Abetract: Shell-model core excitations based on the $\mathrm{Sp}(6, \mathrm{R})$ model are compared with those derived from $\alpha$-cluster models. Overlaps between the first symplectic excitations (essentially giant E2 excitations) and $\alpha$-cluster states of the same SU(3) symmetry are used to estimate the $\alpha$-breakup probabilities of giant E2 resonances in ${ }^{16} \mathrm{O},{ }^{20} \mathrm{Ne},{ }^{24} \mathrm{Mg}$ and ${ }^{28} \mathrm{Si}$. Although these probabilities are large, $\alpha$-cluster and $S p(6, R)$ core excitations are essentially complementary and may both be needed for a meaningful microscopic description of $A=16-40$ nuclei.
\end{abstract}

\section{Introduction}

The symplectic Lie algebra $\operatorname{Sp}(6, R)$ and its subalgebra $\operatorname{Sp}(2, R)$ have recently been shown ${ }^{1,2}$ ) to be useful in selecting the shell-model core excitations needed for the development of quadrupole collective features in a microscopic description of nuclear spectra. These symplectic models are a natural extension of the Elliott SU(3) model, building in core excitations through the development of an SU(3) band superstructure which permits the continuation of rotational bands beyond the simple shell-model limit. Arick ${ }^{2}$ ) has shown that the irreducible representations of the noncompact group $S p(2, R)$ form a meaningful classification scheme for ${ }^{8} \mathrm{Be}$ by comparing the eigenfunctions of an extended shell-model calculation ${ }^{3}$ ) for ${ }^{8} \mathrm{Be}$, including core excitations up to $4 \hbar \omega$, with functions of $\operatorname{good} \operatorname{Sp}(2, R)$ symmetry. A close connection between this $\mathrm{Sp}(2, \mathrm{R})$ model and an $\alpha$-particle model for ${ }^{8} \mathrm{Be}$ has also been demonstrated ${ }^{4}$ ). Since ${ }^{8} \mathrm{Be}$ essentially is two $\alpha$-particles, any meaningful model for ${ }^{8} \mathrm{Be}$ must contain this feature; and it is interesting to investigate whether this close connection between symplectic and $\alpha$-cluster models persists to heavier nuclei. Recent so-called extended shell-model calculations ${ }^{5,6}$ ) have incorporated very high core excitations into the microscopic description of nuclei in the $A=16-44$ region through the introduction of specific $\alpha$-cluster states. The remarkably successful study of ${ }^{16} \mathrm{O}$ by Suzuki $\left.{ }^{7}\right)$ in terms of a pure $\left(\alpha+{ }^{12} \mathrm{C}\right)$ cluster model basis shows that the dominant components of the states in ${ }^{16} \mathrm{O}$ below $16 \mathrm{MeV}$ can seemingly be organized into $\mathrm{SU}(3)$ band systems $\left.^{8}\right)$ of the type $\left(\lambda_{0} \mu_{0}\right),\left(\lambda_{0}+2, \mu_{0}\right),\left(\lambda_{0}+4, \mu_{0}\right), \ldots$, with significant amplitudes up to high values of $\lambda$, and with "bandheads", $\left(\lambda_{0} \mu_{0}\right)$, corresponding to $n \mathrm{p}-n \mathrm{~h}$ states of largest possible intrinsic deformation, with $n=0, \ldots, 5$. Since the $S U(3)$

+ Supported by the US National Science Foundation. 
representations $(\lambda \mu)$ of these band systems are identical with those generated by the infintesimal operators of the noncompact group $\operatorname{Sp}(6, R)$, more specifically the operators of the subgroup $\mathrm{Sp}(2, \mathrm{R})$ which generates intrinsic states of the largest possible deformation, it is interesting to ask how much of the physics of quadrupole excitation is contained in such $\alpha$-cluster model calculations. Both the $\alpha$-cluster model basis and the symplectic excitations select a very specific set of core excited states, free of spurious c.m. motion, from the huge space of shell-model core excitations. It is interesting to determine the extent to which the two types of excitations are identical or complementary. In ${ }^{16} \mathrm{O}$ the overlaps between the first symplectic excitations based on the bandheads $\left(\lambda_{0} \mu_{0}\right)$ with the $\alpha$-cluster states of the same $(\lambda \mu)$ range $\left.{ }^{y}\right)$ from 0.808 to 0.872 . For the second symplectic excitations, however, these overlaps can be as small as 0.549 . In ${ }^{8} \mathrm{Be}$ the corresponding numbers are ${ }^{4}$ ) 0.89 for the first and 0.79 for the second symplectic excitation. In the present work the study of this relationship between $\alpha$-cluster and symplectic excitations is extended to heavier nuclei. Although the $\alpha$-cluster bases contain much of the physics of quadrupole excitations, it is found that the $\alpha$-cluster and symplectic excitations give essentially a complementary set of core excitations, and both may be needed in detailed microscopic studies of nuclei in the $A=16-40$ region.

Since the excitation operators which generate the higher members of a symplectic band are effectively E2 operators, shell-model states of $2 \hbar \omega$ core excitation, corresponding to the first $S p(6, R)$ excitations, can be expected to correspond closely to giant E2 resonances based on the ground state of the system. In nuclei with ground states of relatively pure SU(3) symmetry, states of the first symplectic excitations, with SU(3) labels corresponding to large intrinsic deformations, can be expected to be the dominant components of the giant $\mathrm{E} 2$ resonances of such nuclei. The overlaps between these first $\mathrm{Sp}(6, R)$ excitations and $\alpha$-cluster states of the same $\mathrm{SU}(3)$ symmetry should thus lead to a reliable estimate of the $\alpha$-breakup probability of giant E2 resonances in many light nuclei. Since these overlaps are large, the $\alpha$-breakup channel must be an important contributor to the total width of giant E2 resonances in many light nuclei. The $\alpha$-amplitudes of the first $S p(6, R)$ excitations are given in sect. 3 for a number of $\operatorname{good} \mathrm{SU}(3)$ nuclei ranging from ${ }^{8} \mathrm{Be}$ to ${ }^{28} \mathrm{Si}$.

\section{2. $S p(6, R)$ symmetry}

The symplectic algebra $S p(6, R)$ is generated by the nine $U(3)$ operators

$$
A_{i j}=\frac{1}{2} \sum_{k=1}^{A}\left[\eta_{i}^{\prime+}(k) \eta^{\prime}(k)+\eta^{\prime}(k) \eta_{i}^{\prime+}(k)\right] ; \quad i, j=x, y, z ; \quad k=\text { particle index, }
$$

and the $2 \hbar \omega$ raising and lowering operators

$$
\frac{1}{2} \sum_{k=1}^{A} \eta_{i}^{\prime+}(k) \eta_{j}^{\prime}(k), \quad \frac{1}{2} \sum_{k=1}^{A} \eta^{\prime}(k) \eta_{j}^{\prime}(k),
$$


with SU(3) irreducible tensor character $T^{(20)}$ and $T^{(02)}$. To create excitations free of spurious c.m. motion contamination, the oscillator quantum creation operators, such as

$$
\eta_{x}^{+}(k)=\left[\left(\frac{m \omega}{2 \hbar}\right)^{z} x(k)-i \frac{p_{x}(k)}{(2 \hbar m \omega)^{t}}\right]
$$

must be replaced by

$$
\eta_{i}^{\prime+}(k)=\eta_{i}^{+}(k)-\frac{1}{A} \sum_{l=1}^{A} \eta_{l}^{+}(l)
$$

so that

$$
\begin{gathered}
A_{i j}=\frac{1}{2}\left\{\sum_{k=1}^{A}\left[\eta_{i}^{+}(k) \eta_{j}(k)+\eta_{j}(k) \eta_{i}^{+}(k)\right]-\frac{1}{A} \sum_{l=1}^{A} \sum_{m=1}^{A}\left[\eta_{i}^{+}\left(\eta_{\eta}(m)+\eta(m) \eta_{i}^{+}(l)\right]\right\},\right. \\
T_{i j}^{(20)}=\frac{1}{2}\left\{\sum_{k=1}^{A} \eta_{i}^{+}(k) \eta_{j}^{+}(k)-\frac{1}{A} \sum_{l=1}^{A} \sum_{m=1}^{A} \eta_{l}^{+}(l) \eta_{j}^{+}(m)\right\} \\
T_{i j}^{(02)}=\frac{1}{2}\left\{\sum_{k=1}^{A} \eta_{i}(k) \eta_{j}(k)-\frac{1}{A} \sum_{l=1}^{A} \sum_{m=1}^{A} \eta_{i}\left(l \eta_{j}(m)\right\} .\right.
\end{gathered}
$$

It will also be important to express the $2 \hbar \omega$ raising operators is (properly normalized) irreducible tensor operators $T_{s A A_{A}}^{(20)}$ in the $\mathrm{SU}(3) \supset \mathrm{SU}(2) \times \mathrm{U}(1)$ basis, characterized by the Elliott quantum numbers ${ }^{9}$ ) $\varepsilon \Lambda M_{A}$ :

$$
\begin{array}{cl}
T_{z z}^{(20)}=T_{400}^{(20)}, & T_{x x}^{(20)}=T_{-21 \pm 1}^{(20)}, \\
T_{x z}^{(20)}=\sqrt{\frac{1}{2}} T_{1 \pm \pm \pm}^{(20)}, & T_{x y}^{(20)}=\sqrt{\frac{1}{2}} T_{-210}^{(20)},
\end{array}
$$

and, finally, in terms of the polynomials $\left.{ }^{10}\right) P_{a}^{(20)}\left(\eta^{+}\right)$in the harmonic oscillator creation operators

$$
T_{\alpha}^{(20)}=\frac{1}{2}\left\{\sqrt{2} \sum_{k=1}^{A} P_{\alpha}^{(20)}\left(\eta^{+}(k)\right)-\frac{1}{A} \sum_{l, m=1}^{A}\left[P^{(10)}\left(\eta^{+}(l)\right) \times P^{(10)}\left(\eta^{+}(m)\right)\right]_{\alpha}^{(20)}\right\},
$$

where $P_{\alpha}^{(20)}$ creates a normalized oscillator function, when acting on the oscillator ground state. The square bracket denotes $\mathrm{SU}(3)$ coupling, and $\alpha$ is any component of the (20) tensor in either the $\varepsilon A M_{A}$ or the $L M_{L}$ scheme.

Earlier applications ${ }^{11,12}$ ) of this algebra have made elegant use of $S p(6, R)$ symmetry in the space of harmonic oscillator functions of a single (three-dimensional) variable, but have missed the rich spectrum of symplectic bands in a many-nucleon system. The important implications of the existence of this spectrum have only recently been recognized by Rosensteel and Rowe $\left.{ }^{1}\right)^{\dagger}$. The U(3) quantum numbers of

+ These authors use the notation $\operatorname{Sp}(3, \mathbf{R})$ in place of the more conventional $\mathrm{Sp}(6, \mathbf{R})$. 
any 'ralence configuration (states of 0 hw oscillator excitation) characterize an (infinite-dimensional) unitary irreducible representation of the noncompact group $\operatorname{Sp}(6, R)$. The three labels $\lambda_{0} \mu_{0} v_{0}$ will be chosen to characterize these representations, where $\left(\lambda_{0} \mu_{0}\right)$ are the Elliott SU(3) quantum numbers of the symplectic bandhead, while the $U(3)$ quantum numbers of the bandhead, $\lambda_{0}+\mu_{0}+v_{0}, \mu_{0}+v_{0}$, and $v_{0}$ give the total number of oscillator quanta in the three space directions. Certain core excited states can form further symplectic bandheads. The $n p$-nh states of largest possible intrinsic deformation, such as the $4 \mathrm{p}-4 \mathrm{~h}$ state in ${ }^{16} \mathrm{O}$ with $(\lambda \mu)=(84)$, e.g. characterize further irreps of $\operatorname{Sp}(6, R)$. (The lowering operators $T^{(02)}$ must annihilate this state, since (02) operators acting on this (84) state could create only states with $(\lambda \mu)=(10,2)$, (64) (86), (83), (75) and (94). No such Pauli-allowed states exist in the space of core excitations of $2 \hbar \omega$.

The matrix elements of the $2 \hbar \omega$ raising operators with $l=2$ are proportional to the matrix elements of the mass quadrupole operator (defined relative to the c.m. of the system). Between SU(4) scalar states they are also proportional to the matrix elements of the charge quadrupole operator; and for this reason nuclei with $A=4 n$ in the $A \leqq 28$ region will be chosen as the prime examples. For giant E2 excitations based on ground states the first symplectic excitations are of greatest interest. They are given by

$$
\left[T^{(20)} \times \Psi^{\left(\lambda_{0} \mu_{0}\right)}\right]_{a}^{(\lambda \mu)},
$$

where $\Psi^{\left(\lambda_{0} m_{0}\right)}$ is the symplectic bandhead wave function, which is assumed to be the major SU(3) component of the actual $A$-nucleon ground-state wave function; and where the square bracket again denotes SU(3) coupling. E.g.

$$
\begin{aligned}
\Psi_{\kappa L M}^{(\lambda \mu)}=\left[T^{(20)} \times \Psi^{\left(\lambda_{0} \mu_{0}\right)}\right]_{\kappa L M}^{(\lambda \mu)}\left[\left\langle(\lambda \mu)\left\|T^{(20)}\right\|\left(\lambda_{0} \mu_{0}\right)\right\rangle\right]^{-1} \\
=\sum_{l x_{0} L_{0} m M_{0}}\left\langle(20) l ; ;\left(\lambda_{0} \mu_{0}\right) \kappa_{0} L_{0} \|(\lambda \mu) \kappa L M\right\rangle\left\langle m L_{0} M_{0} \mid L M\right\rangle \\
\times\left[\left\langle(\lambda \mu)\left\|T^{(20)}\right\|\left(\lambda_{0} \mu_{0}\right)\right\rangle\right]^{-1} T_{l m}^{(20)} \Psi_{\kappa_{0} L_{0} M_{0}}^{\left(\lambda_{0}\left(\mu_{0}\right)\right.}
\end{aligned}
$$

given here in the angular momentum basis, $\alpha=\kappa L M$, so that the reduced Wigner coefficient needed here is an SU(3)/R(3) reduced coefficient. With $\alpha=\varepsilon \Lambda M_{A}$ the

\section{TABLE 1}

Reduced matrix elements of the first $S p(6, R)$ excitations in the band characterized by $\lambda_{0} \mu_{0} v_{0}$

\begin{tabular}{ll}
\hline \multicolumn{1}{c}{$\left(\lambda^{\prime} \mu^{\prime}\right)$} & $\left.K\left(\lambda^{\prime} \mu^{\prime}\right)\left\|T^{(20)}\right\|\left(\lambda_{0} \mu_{0}\right)\right\rangle\left.\right|^{2}$ \\
\hline$\left(\lambda_{0}+2, \mu_{0}\right)$ & $\frac{1}{2}\left[2\left(\lambda_{0}+\mu_{0}+v_{0}\right)+A-1\right]$ \\
$\left(\lambda_{0}, \mu_{0}+1\right)$ & $\frac{1}{2}\left[\lambda_{0}+2 \mu_{0}+2 v_{0}+A-3\right]$ \\
$\left(\lambda_{0}+1, \mu_{0}-1\right)$ & $\frac{1}{2}\left[\lambda_{0}+\mu_{0}+2 v_{0}+A-4\right]$ \\
$\left(\lambda_{0}-2, \mu_{0}+2\right)$ & $\frac{1}{2}\left[2\left(\mu_{0}+v_{0}\right)+A-3\right]$ \\
$\left(\lambda_{0}-1, \mu_{0}\right)$ & $\frac{1}{2}\left[\mu_{0}+2 v_{0}+A-5\right]$ \\
$\left(\lambda_{0}, \mu_{0}-2\right)$ & $\frac{1}{2}\left[2 v_{0}+A-5\right]$ \\
\hline
\end{tabular}


Wigner coefficients must simply be replaced with the appropriate coefficients for the $\mathrm{SU}(3) \supset \mathrm{SU}(2) \times \mathrm{U}(1)$ basis.

For these first symplectic excitations the states $(\lambda \mu)$ are simple, (have a single occurrence in a symplectic band). The SU(3) reduced matrix elements of $T^{(20)}$ for these first symplectic excitations are given in table 1 . Another set of simple states within the symplectic band are the states of largest possible intrinsic deformation for each core excitation of $2 m \hbar \omega$ which form the SU(3) ladder $\left(\lambda_{0} \mu_{0}\right),\left(\lambda_{0}+2, \mu_{0}\right), \ldots,\left(\lambda_{0}+2 m\right.$, $\left.\mu_{0}\right), \ldots$..To generate these states it is sufficient to consider the subalgebra $\left.{ }^{2}\right) \operatorname{Sp}(2, \mathbf{R})$ generated by $F_{+}=T_{z z}^{(20)}, F_{-}=T_{z z}^{(02)}$, and $F_{0}=\frac{1}{2} A_{z x}$ Even though this subalgebra does not contain the angular momentum operators, it is sufficient to create states of the form (7) with $(\lambda \mu)=\left(\lambda_{0}+2 m, \mu_{0}\right)$ and $\alpha=\kappa L M$, since we can use the WignerEckart theorem, as expressed by eq. (8), and since the SU(3)-reduced matrix elements of $T^{(20)}$ for the full $\mathrm{Sp}(6, \mathrm{R})$ group are given by matrix elements of $T_{\mathrm{zz}}^{(20)}$ between highest weight states, $\mathrm{HW}$, in the $\varepsilon \Lambda M_{A}$ scheme

$$
\begin{aligned}
& \left\langle\left(\lambda_{0}+2 m+2, \mu_{0}\right) \mathrm{HW}\left|T_{x z}^{(20)}\right|\left(\lambda_{0}+2 m, \mu_{0}\right) H W\right\rangle \\
& \quad=1 \times\left\langle\left(\lambda_{0}+2 m+2, \dot{\mu}_{0}\right)\left\|T^{(20)}\right\|\left(\lambda_{0}+2 m, \mu_{0}\right)\right\rangle=-[(m+1)(m+2|F|)]^{ \pm},
\end{aligned}
$$

with $m=0,1,2 \ldots$, where $|F|$ is given by the minimum possible eigenvalue of $F_{0}$ (from table 1, 2|F|= $\lambda_{0}+\mu_{0}+v_{0}+\frac{1}{2}(A-1)$ ).

To give the actual shell-model decomposition of a symplectic excitation, it is useful to express the $2 \hbar \omega$-raising operator in (7) in terms of the polynomials $P^{(q)}\left(\eta^{+}\right)$of eq. (6), since the shell-model decomposition of SU(3) coupled states of this form can readily be evaluated by $\mathrm{SU}(3)$, SU(4) recoupling techniques of the type used to give the shell-model decomposition of $\alpha$-cluster states in refs. ${ }^{13}$. 14). Table 2 gives the shellmodel components of the first symplectic excitation with $(\lambda \mu)=(10,0)$, based on the ${ }^{20} \mathrm{Ne} 0 \hbar \omega$ excitation state with $\left(\lambda_{0} \mu_{0}\right)=(80),\left(v_{0}=4\right)$, and for comparison the $\alpha$-clusster state

$$
N_{\left(\lambda_{\mu}\right)}^{Q}\left[\phi^{\left(\lambda_{c} H_{c}\right)=(00)}\left({ }^{16} \mathrm{O}\right) \times \phi^{(00)}(\alpha) \times \Phi_{\left(r_{e}-100\right)}^{(00)}\right]_{L H)}^{\left(\lambda_{\mu}\right)=(Q 0)},
$$

with a harmonic $\alpha-{ }^{16} \mathrm{O}$ relative motion function, carrying $Q=10$ oscillator quanta, and ${ }^{16} \mathrm{O}$ core and $\alpha$-particle closed shell internal wave functions, $\phi$, with $(\lambda \mu)=(00)$. (In eq. (10) $A$ is the antisymmetrizer and $N$ the norm factor.)

The large amplitude components of the $\operatorname{Sp}(6, R)$ state (the first three entries of table 2) are those in which a single particle is excited by $2 \hbar \omega$, up from the sd to the sdg shell, or from the $\mathrm{p}$ to the $\mathrm{pf}$ shell (without change in the sd ${ }^{4}$ configuration). The four small components, making up only $2 \%$ of the $\operatorname{Sp}(6, \mathbf{R})$ wave function, involve simultaneous excitations of two particles by $1 \mathrm{~h} \omega$. These components are generated by the $1 / A$ parts of the $2 h \omega$-raising operators, and are needed to insure a final state free of spurious c.m. excitations. The $\alpha$-cluster state also has large components in which a single particle is excited by $2 \hbar \omega$. but the similarity between the two types of states is now less pronounced than it is for similar excitations ${ }^{8}$ ) in ${ }^{16} \mathrm{O}$ and ${ }^{8} \mathrm{Be}$. The overlap between the $(10,0) \mathrm{Sp}(6, \mathrm{R})$ and $\alpha$-cluster states in ${ }^{20} \mathrm{Ne}$ is 0.686 , which compares $\left.{ }^{8,4}\right)$ with overlaps 
TABLE 2

Shell-model decomposition of the $\operatorname{Sp}(6, \mathrm{R})$ and $\alpha$-cluster states with $(\lambda \mu)=(10,0)$ in ${ }^{20} \mathrm{Ne}$

\begin{tabular}{lrr}
\hline & \multicolumn{2}{c}{ Amplitudes } \\
\cline { 2 - 3 } Shell-model component $\left.{ }^{*}\right)$ & $\mathrm{Sp}(6, \mathrm{R})$ & $\alpha$-cluster \\
\hline$\left|\mathrm{s}^{4} \mathrm{p}^{12}\left[\mathrm{sd}^{3} \mathrm{sdg}^{1}\right](10,0)\right\rangle$ & 0.710 & 0.505 \\
$\left|\mathrm{~s}^{4}\left[\mathrm{p}^{11}(01)\left[\mathrm{sd}^{4}(80) \mathrm{pf}^{1}\right](11,0)\right](10,0)\right\rangle$ & 0.603 & 0.494 \\
$\left|\mathrm{~s}^{4}\left[\mathrm{p}^{11}(01)\left[\mathrm{sd}^{4}(80) \mathrm{pf}^{1}\right](91)\right](10,0)\right\rangle$ & 0.336 & 0.068 \\
$\left|\mathrm{~s}^{4}\left[\mathrm{p}^{11}(01)\left[\mathrm{sd}^{4}(61) \mathrm{pf}^{1}\right](91)\right](10,0)\right\rangle$ & 0.102 & 0.503 \\
$\left|\mathrm{~s}^{4} \mathrm{p}^{12}\left[\mathrm{sd}^{2} \mathrm{pf}^{2}\right](10,0)\right\rangle$ & -0.079 & 0.260 \\
$\left|\mathrm{~s}^{4}\left[\mathrm{p}^{10}(02) \mathrm{sd}^{6}(82)\right](10,0)\right\rangle$ & 0.046 & -0.337 \\
$\left|\mathrm{~s}^{4}\left[\mathrm{p}^{10}(10) \mathrm{sd}^{6}(90)\right](10,0)\right\rangle$ & 0.034 & -0.251 \\
\hline
\end{tabular}

2) The shell-model components are given in SU(3), SU(4) coupled form. Square brackets denote both SU(3) and SU(4) coupling. Redundant quantum numbers are omitted. Since all SU(4) quantum numbers follow from the SU(3) labels $(\lambda \mu)$ they are not shown explicitly.

of 0.808 and 0.894 for the corresponding states in ${ }^{16} \mathrm{O}$, with $(\lambda \mu)=(20)$, and ${ }^{8} \mathrm{Be}$ with $(\lambda \mu)=(60)$. To study the relationship between the $\alpha$-cluster model and the $S p(6, R)$ excitations further, higher excitations in the symplectic ladder, $\left(\lambda_{0}+2 m, \mu_{0}\right)$, of ${ }^{20} \mathrm{Ne}$ are compared with the corresponding $\alpha$-cluster states with $\alpha-{ }^{16} \mathrm{O}$ relative motion function carrying $Q=\lambda_{0}+2 m$ quanta. The overlaps between the two types of states are shown in table 3. [Norm factors for the $\alpha$-cluster states have been given by Bandō $\left.{ }^{6}\right)$, the normalizations for the $\operatorname{Sp}(6, R)$ states follow from eq. (9).] Although there is sufficient overlap between the first symplectic excitation and the corresponding $\alpha$-cluster state to give a large $\alpha$-breakup probability to an E2 excitation based on the (80) ${ }^{20} \mathrm{Ne}$ shell-model valence configuration, the higher symplectic excitations diverge more and more from the corresponding $\alpha$-cluster states. The higher symplectic excitations which are important for the full development of the quadrupole collective features of the ${ }^{20} \mathrm{Ne}$ spectrum are thus largely complementary to the $\alpha$-cluster excitations of the same SU(3) symmetry. [A similar conclusion for ${ }^{20} \mathrm{Ne}$ was reached by Bando ${ }^{6}$ ) with a successful application of a mixed $\alpha$-cluster and E2 excitation model, however without the use of $S p(6, R)$ symmetry.]

The symplectic excitations also contain $(\lambda \mu)$ which are not found in the $\alpha$-cluster basis but which must play a role in the incorporation of quadrupole collective features

TABLE 3

Overlaps between sympletic excitations with $\left(\lambda_{\mu}\right)=\left(\lambda_{0}+2 m, 0\right)$ and $\alpha$-cluster states in ${ }^{20} \mathrm{Ne}$

\begin{tabular}{cc}
\hline$(\lambda \mu)$ & Overlap \\
\hline$(80)$ & 1 \\
$(10,0)$ & 0.686 \\
$(12,0)$ & 0.495 \\
$(14,0)$ & 0.364 \\
$(16,0)$ & 0.281 \\
\hline
\end{tabular}


TABLE

Shell-model decompositions of the first $\operatorname{Sp}(6, \mathbf{R})$ excitations in

Shell-model component ")

$$
\begin{aligned}
& \underline{(\lambda \mu)=(81)} \\
& \left|s^{4} p^{12}\left[\mathrm{sd}^{3}(60) \mathrm{sdg}^{1}(40)\right](81)\right\rangle \\
& \left|\left[\mathrm{s}^{3} \mathrm{p}^{12} \mathrm{sd}^{3}(81)\right](81)\right\rangle \\
& \left|s^{4} p^{12}\left[s d^{2}(40) p f^{2}(60)\right](81)\right\rangle \\
& \left.\left|s^{4}\left[p^{11}(01)\left[s d^{4}\left[f_{4}\right]=[0]\left(\lambda_{4} \mu_{4}\right) \mathrm{pf}^{1}\right]\left(\lambda_{3} \mu_{5}\right)\right](81)\right\rangle^{b}\right) \\
& \left.\left|\mathrm{s}^{4}\left[\mathrm{p}^{11}(01)\left[\mathrm{sd}^{4}\left[\mathrm{f}_{4}\right]=[211]\left(\lambda_{4} \mu_{4}\right) \mathrm{pf}^{1}\right]\left(\lambda_{3} \mu_{5}\right)\right](81)\right\rangle^{b}\right) \\
& \left|s^{4}\left[p^{10}\left(\lambda_{10} \mu_{10}\right) s d^{6}\left(\lambda_{6} \mu_{6}\right)\right](81)\right\rangle \\
& \underline{(\lambda \mu)=(62)} \\
& \left|\left[s^{3} p^{12} s d^{5}(62)\right](62)\right\rangle \\
& \left.\mid s^{4} p^{12}\left[\operatorname{sd}^{3}(60) \text { sdg }^{1}(40)\right](62)\right\rangle \\
& \left|s^{4} p^{12}\left[s d^{2}(40) p f^{2}(60)\right](62)\right\rangle \\
& \left|s^{4} \mathrm{p}^{12}\left[\mathrm{sd}^{2}(40) \mathrm{pf}^{2}(22)\right](62)\right\rangle \\
& \left.\left.\left|s^{4}\left[p^{11}(01)\left[s d^{4}[0]\left(\lambda_{4} \mu_{4}\right) p f^{1}\right]\left(\lambda_{s} \mu_{s}\right)\right](62)\right\rangle\right\rangle^{b}\right) \\
& \left.\left.\left|s^{4}\left[p^{11}(01)\left[8 d^{4}[211]\left(\lambda_{4} \mu_{4}\right) \mathrm{pf}^{1}\right]\left(\lambda_{3} \mu_{3}\right)\right](62)\right\rangle\right\rangle^{b}\right) \\
& \left.\left|s^{4}\left[p^{10}(02) s d^{6}\left(\lambda_{6} \mu_{6}\right),\right](62)\right\rangle^{c}\right) \\
& \left.\left|s^{4}\left[p^{10}(10) s d^{6}\left(\lambda_{6} \mu_{6}\right)_{l}\right](62)\right\rangle^{9}\right)
\end{aligned}
$$

-) For notation, see table 2.

b) In these cases SU(4) quantum numbers do not always follow from $\left(\lambda_{4} \mu_{4}\right)$, and $\left[f_{4}\right]$ is indicated.

c) In the case of multiple occurrences of $\left(\lambda_{6} \mu_{6}\right)$ within $\left[f_{6}\right]$, states are chosen according to refs. $\left.{ }^{15}, 16\right)$. All zero amplitudes.

into the microscopic description of the spectrum. In the case of the first symplectic excitations for ${ }^{20} \mathrm{Ne}$, these are the states with $(\lambda \mu)=(81)$ and $(62)$. The shell-model decompositions for these symplectic excitations are shown in table 4 . In both states there are only four large shell-model components, corresponding to excitations of a single particle by $2 \hbar \omega$, from the $s$ to the sd shell, from the $p$ to the pf shell, and from the sd to the sdg shell. The small components, comprising $2.81 \%$ of the wave function for $(\lambda \mu)=(81)$, and $3.69 \%$ for $(\lambda \mu)=(62)$, are again generated by the $1 / A$ parts of the $2 h \omega$-raising operators. They are included in table 4 mainly to illustrate the rich number of possibilities for core excitations of $2 h \omega$, even when restricted to a specific SU(3) representation $(\lambda \mu)$. The symplectic excitations select a very specific set of states out of the large shell-model space of core excitations. 
${ }^{20} \mathrm{Ne}$ with $(\lambda \mu)=(81)$ and $(62)$

Amplitude

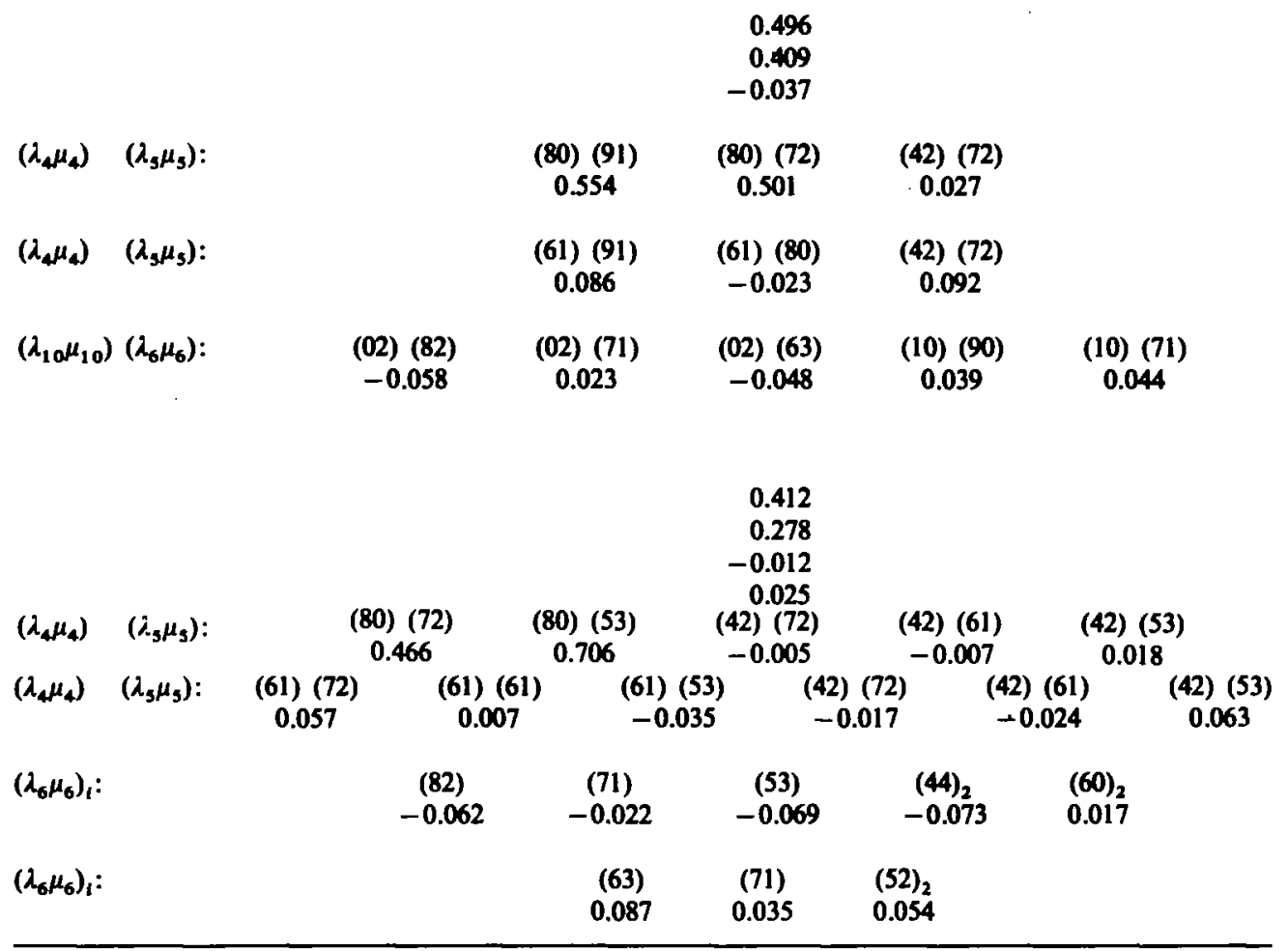

needed sd shell c.f.p. follow from refs. ${ }^{15.16}$ ). In the three cases with multiple occurrences, states with $i=1$ have

For the higher excitations of table 3 and for heavier nuclei, the full shell-model decompositions would involve a huge number of shell model components. Overlaps between the $S p(6, R)$ and $\alpha$-cluster states can, however, be evaluated very simply with the use of so-called "cluster-like" functions ${ }^{17,13}$ ) in which the relative motion oscillator function $\Phi^{(\ell)}\left(r_{\varepsilon-c}\right)$ of eq. (10) is replaced by a function of the $\alpha$-particle c.m. vector, $\Phi^{(20)}\left(R_{a}\right)$. Since the $\operatorname{Sp}(6, R)$ states are rigorously free of spurious c.m. motion excitations, the spurious components of $\Phi^{(Q)}\left(R_{\alpha}\right)$ cannot make a contribution to the overlaps. Since ${ }^{13}$ )

$$
\Phi^{(Q 0)}\left(R_{a}\right)=\left(\frac{A-4}{A}\right)^{+Q} \Phi^{(Q 0)}\left(r_{a-c}\right)+\ldots
$$


where $+\ldots$ contains only pieces with spurious c.m. motion excitations, the overlap between a $S p(6, R)$ state and a true cluster function is equal to $[A /(A-4)]^{t Q} \times$ (overlap between the $S p(6, R)$ state and the corresponding "cluster-like" function). To calculate the latter overlap, projection onto a small number of "cluster-like" shell-model components is sufficient; the "cluster-like" shell-model components being those in which the shell-model configurations and SU(3) quantum numbers are those of the $(A-4)$-particle core wave function, in the case of ${ }^{20} \mathrm{Ne}$ only shell-model components of the type $\left|\mathbf{s}^{4} \mathrm{p}^{12}(00)(\mathrm{sd}, \mathrm{pf}, \ldots)^{4}(Q 0)\right\rangle$. (Of the seven shell-model components of table 2, only the first and fifth are "cluster-like".) For the validity of this technique, however, it is important that the $\operatorname{Sp}(6, R)$ states are rigorously free of spurious c.m. contaminations. The four small components in the $\operatorname{Sp}(6, R)$ wave function of table 2 which insure this property make up only $2 \%$ of the wave function. The neglect of these small components, however, especially when propagated to the higher symplectic excitations, can lead to errors $Z 50 \%$ in the evaluation of overlaps or matrix elements. The $1 / A$ parts of the $2 \hbar \omega$-raising operators can thus not be neglected for nuclei in the $A \approx 20$ mass range.

Overlaps between $\alpha$-cluster states and the first symplectic excitations for some heavier nuclei are included in table 5. These overlaps decrease with $A$ and in general are largest for the SU(3) excitations corresponding to largest possible intrinsic deformations.

TABLE 5

Overlaps between $\alpha$-cluster states and the first $\operatorname{Sp}(6, R)$ excitations in $A=4 n$ nuclei

\begin{tabular}{lcclll}
\hline Nucleus & $\begin{array}{c}\text { Symplectic } \\
\text { bandhead }\left(\lambda_{0} \mu_{0}\right)\end{array}$ & $\begin{array}{c}(A-4) \text {-cluster } \\
\text { core }\left(\lambda_{\mathrm{c}} \mu_{\mathrm{c}}\right)\end{array}$ & \multicolumn{1}{c}{$(\lambda \mu)$} & Overlap & $\begin{array}{c}\alpha \text {-breakup } \\
\text { factors }{ }^{0}\end{array}$ \\
\hline${ }^{8} \mathrm{Be}$ & $(40)$ & $(00)$ & $(60)$ & 0.894 & 1.095 \\
& & & $(41),(22)$ & 0 & 0 \\
${ }^{16} \mathrm{O}$ & $(00)$ & $(04)$ & $(20)$ & 0.808 & 0.950 \\
${ }^{20} \mathrm{Ne}$ & $(80)$ & $(00)$ & $(10,0)$ & 0.686 & 0.490 \\
& & & $(81),(62)$ & 0 & 0 \\
${ }^{24} \mathrm{Mg}$ & $(84)$ & $(80)$ & $(10,4)$ & 0.599 & 0.448 \\
& & & $(85)$ & 0.612 & 0.387 \\
& & & $(66)$ & 0.260 & 0.183 \\
${ }^{28} \mathrm{Si}$ & $(0,12)$ & $(84)$ & $(2,12)$ & 0 & 0 \\
& & & $(1,11)$ & 0.721 & 0.608 \\
& & & $(0,10)$ & 0.319 & 0.212 \\
& & & & 0.084 & 0.058 \\
\hline
\end{tabular}

9) The $x$-breakup factors are $\left(1 / N_{(2, \mathrm{~m}}^{0}\right) \times$ overlap, where the overlaps are between normalized $\alpha$-cluster and $\operatorname{Sp}(6, R)$ states.

\section{The $\alpha$-break up of giant $\mathbf{E 2}$ resonances in light nuclel}

The overlaps between the $\alpha$-cluster and $\operatorname{Sp}(6, R)$ states calculated in sect. 2 can lead to reasonable estimates of the $\alpha$-breakup probabilities of giant E2 resonances in 
nuclei with ground states of relatively pure SU(3) symmetry. In such nuclei the first symplectic excitations can be expected to be major components of the giant E2 resonances. This approximation should be particularly good in $A=4 n$ nuclei with predominant SU(4) scalar character, for which the matrix elements of both charge and mass quadrupole operators are proportional to the matrix elements of the $2 h \omega$-raising operators of the symplectic algebra. Even in such nuclei we can distinguish two extremes, the SU(3) strong and weak coupling approximations. In the SU(3) strong coupling approximation, it is assumed that the dominant component of the giant E2 excitation has good SU(3) symmetry and is given by the SU(3) state $(i \mu)$ from the first symplectic excitations, $\left[\left(\lambda_{0} \mu_{0}\right) \times(20)\right] \rightarrow(\lambda \mu)$, corresponding to largest possible intrinsic deformation, normally the state with $(\lambda \mu)=\left(\lambda_{0}+2, \mu_{0}\right)$ (a normalized SU(3) coupled state of the form of eq. (8)). In this approximation the $x$-amplitude ${ }^{17.13}$ ) follows from the simple overlaps (table 5)

$$
\begin{aligned}
A_{J=2+\rightarrow 0^{+}}=\left\langle\left(\lambda_{\mathrm{c}} \mu_{\mathrm{c}}\right) \kappa_{\mathrm{c}}=0 L_{\mathrm{c}}=0\right. & ;(Q 0) L=2 \|(\lambda \mu) \kappa 2\rangle \\
& \times \frac{1}{N_{(\lambda \mu)}^{Q}}\left\langle\Psi(\operatorname{Sp}(6, \mathrm{R}))^{(\lambda \mu) \mid \Psi\left(\left(\lambda_{\mathrm{c}} \mu_{\mathrm{c}}\right) \times(Q 0)(\lambda \mu)\right.}\right\rangle,
\end{aligned}
$$

where the double-barred coefficient is an $\mathrm{SU}(3) / R(3)$ Wigner coefficient which can be obtained from the code of Akiyama and Draayer ${ }^{18}$ ). The overlap is between the normalized $S p(6, R)$ excitations and the $\alpha$-cluster state of eq. (10), where it is assumed that the $(A-4)$-particle daughter nucleus has a ground state of good SU(3) symmetry $\left(i_{c} \mu_{\mathrm{c}}\right)$.

In the weak-coupling approximation the dominant component of the giant E2 excitation is assumed to have the form

$$
1\left[T_{I=2}^{(20)} \times \Psi_{\mathrm{k}_{0} L_{0} S_{0} J_{0}}^{\left(\lambda_{0 \mu}\right)}\right]_{J M}
$$

\begin{tabular}{|c|c|c|c|}
\hline \multirow[b]{2}{*}{ Nucleus ") } & \multirow{2}{*}{ Strong coupling $(\lambda \mu)$} & \multicolumn{2}{|c|}{$\boldsymbol{x}$-amplitudes } \\
\hline & & $\begin{array}{l}\text { strong-coupling }{ }^{\text {b }} \text { ) } \\
\text { approximation }\end{array}$ & $\begin{array}{c}\text { weak-coupling ') } \\
\text { approximation }\end{array}$ \\
\hline${ }^{16} \mathrm{O}$ & (20) & 0.233 & $\left.0.233^{\star}\right)$ \\
\hline${ }^{20} \mathrm{Ne}$ & $(10,0)$ & 0.490 & 0.260 \\
\hline${ }^{2+} \mathbf{M g}$ & $(10,4)$ & $\begin{array}{l}\kappa=0: 0.146 \\
\kappa=2: 0.028\end{array}$ & 0.136 \\
\hline${ }^{28} \mathrm{Si}$ & $(2,12)$ & $\begin{array}{l}\kappa=0: 0.093 \\
\kappa=2: 0.127\end{array}$ & 0.129 \\
\hline
\end{tabular}

TABLE 6

The $x$-breakup amplitudes for $2^{+}$E2 excitations into daughters in $\mathbf{0}^{+}$ground states

") The symplectic bandhead $\left(\lambda_{0} \mu_{0}\right)$ and $(A-4)$-cluster daughter $\left(\lambda_{c} \mu_{c}\right)$ are those given in table 5 .

b) See eq. (12).

c) See eq. (14).

d) In ${ }^{16} \mathrm{O}$ there is no distinction between strong and weak coupling approximations. 
where the square bracket now denotes angular momentum coupling only; so that the state is a mixture of all $(\lambda \mu)$ of the first $\operatorname{Sp}(6, R)$ excitations $\left[\left(\lambda_{0} \mu_{0}\right) \times(20)\right]$, although $\left(\lambda_{0} \mu_{0}\right)$ is assumed to be a good quantum number. (The norm factor, $\mathcal{N}$, follows from the reduced matrix elements of table 1 and SU(3)/R(3) Wigner coefficients.) In this weak-coupling approximation the $\alpha$-amplitude has the form

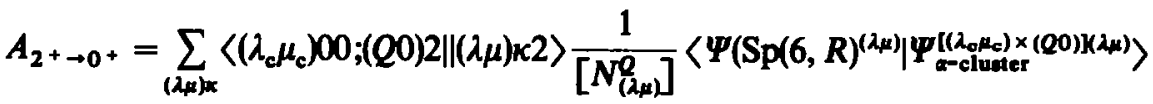

$$
\begin{aligned}
& \times \frac{\left\langle(\lambda \mu)\left\|T_{0}^{(20)}\right\|\left(\lambda_{0} \mu_{0}\right)\right\rangle\left\langle\left(\lambda_{0} \mu_{0}\right) 00 ;(20) 2 \|(\lambda \mu) \kappa 2\right\rangle}{\left[\sum_{\left(\lambda^{\prime} \mu^{\prime}\right) \kappa^{\prime}}\left\langle\left(\lambda_{0} \mu_{0}\right) 00 ;(20) 2 \|\left(\lambda^{\prime} \mu^{\prime}\right) \kappa^{\prime} 2\right\rangle^{2} \times\left\langle\left(\lambda^{\prime} \mu^{\prime}\right)\left\|T^{(20)}\right\|\left(\lambda_{0} \mu_{0}\right)\right\rangle^{2}\right]^{2}} .
\end{aligned}
$$

Some examples are given in table 6. For comparison it may be useful to recall the SU(3) shell-model estimate for the $\alpha$-amplitude of the ${ }^{20} \mathrm{Ne}$ ground state ${ }^{17}$ ), $A=0.48$. The $\alpha$-breakup channel can thus be expected to be a significant contributor to the total width of giant E2 resonances; and the $\alpha$-amplitudes of table 6 must be taken into consideration ${ }^{19}$ ) in the study of the giant E2 resonances recently observed ${ }^{20}$ ) in these nuclei.

Although there is sufficient overlap between the first $\mathrm{Sp}(6, R)$ excitations and the corresponding $\alpha$-cluster states to give giant E2 excitations a large $\alpha$-breakup probability; we conclude that $\alpha$-cluster and symplectic excitations are essentially complementary in all but the lightest nuclei. In order to incorporate both the physics of quadrupole collectivity and $\alpha$-clustering, extended shell-model calculations should include core excitations of both types.

\section{References}

1) G. Rosensteel and D. J. Rowe, Phys. Rev. Lett. 38 (1977) 10

2) F. Arickx, Nucl. Phys. A268 (1976) 347

3) F. Arickx, P. van Leuven and M. Bouten, Nucl. Phys. A252 (1975) 416

4) F. Arickx, Nucl. Phys. A284 (1977) 264

5) T. Tomoda and A. Arima, Proc. Int. Conf. on cluster structure of nuclei and transfer reactions induced by heavy ions, Tokyo, 1975;

A. Arima, Proc. 2nd Int. Conf. on clustering phenomena in nuclei, College Park, Maryland, 1975

6) H. Bando, Nucl. Phys. A261 (1976) 269

7) Y. Suzuki, Prog. Theor. Phys. 55 (1976) 1751 ; 56 (1976) 111

8) K. T. Hecht, Contribution to Int. Conf. on nuclear structure, Tokyo, 1977

9) J. P. Elliott, Proc. Roy. Soc. A245 (1958) 128; 562

10) P. Kramer and D. Schenzle, Nucl. Phys. A204 (1973) 593;

T. A. Brody and M. Moshinsky, Rev. Mex. Fis. 9 (1960) 181

11) C. Quesne and M. Moshinsky, J. Math. Phys. 12 (1971) 1780

12) B. G. Wybourne, Classical groups for physicists (Wiley, NY, 1974)

13) K. T. Hecht, Nucl. Phys. A283 (1977) 223

14) K. T. Hecht, to be published in Phys. Rev. $\bar{C}$

15) K. T. Hecht and D. Braunschweig, Nucl. Phys. A244 (1975) 365

16) D. Braunschweig, computer code to be published in Comp. Phys. Commun.

17) M. Ichimura, A. Arima, E. C. Halbert and T. Terasawa, Nucl. Phys. A204 (1973) 225

18) Y. Akiyama and J. P. Draayer, Comp. Phys. Commun. 5 (1973) 405

19) A. Faessler, Private communication

20) K. T. Knőpfle, G. J. Wagner, H. Breuer, M. Rogge and C. Mayer-Böricke, Phys. Rev. Lett. 35 (1975) 779 ;

K. T. Knōpfle, G. J. Wagner, A. Kiss, M. Rogge and C. Mayer-Bőricke, Phys. Lett. 64B (1976) 263 